Abstract 457 Table 1 Comparing the frequency of Revised ACR Classification Criteria for SLE Identified Two Databases, CLD (disease specific) and NMEDW (EHR).

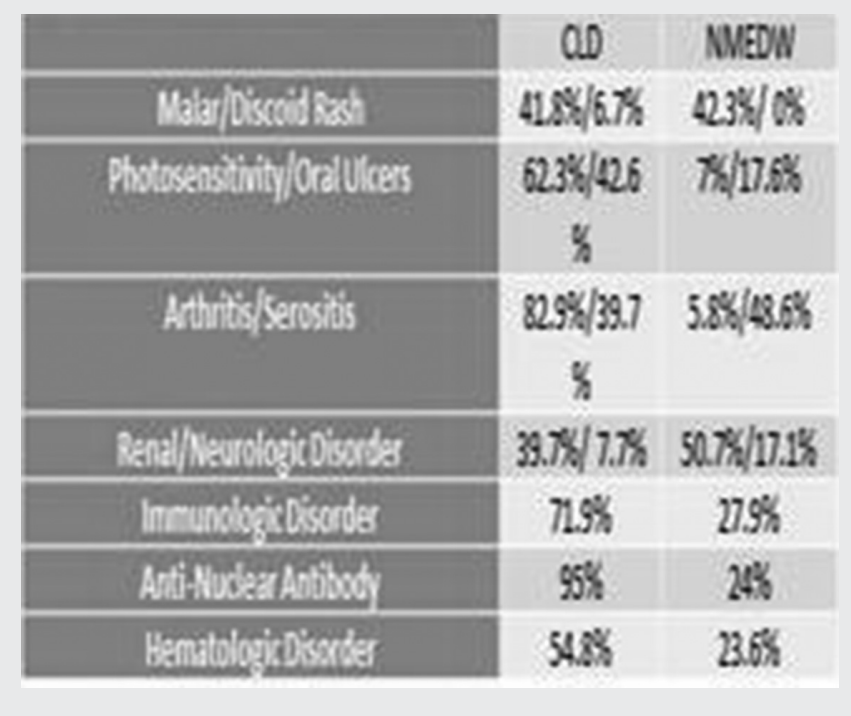

\section{INCREASED ADHESION MOLECULE LEVELS IN SYSTEMIC LUPUS ERYTHEMATOSUS: RELATIONSHIPS WITH SEVERITY OF ILLNESS, AUTOIMMUNITY AND CORTISOL LEVELS}

${ }^{1}$ A Name Colado Simão*, ${ }^{2}$ L Flor da Rosa Santos Silva, ${ }^{1}$ MA Batisti Lozovoy, ${ }^{3}$ B Miglioranza Scavuzzi, ${ }^{2} \mathrm{~N}$ Perugini Stadtlober, ${ }^{4} \mathrm{~T}$ Veiga Mayumi Iriyoda, ${ }^{1} \mathrm{EM}$ Vissoci Reiche, ${ }^{5}$ I Dichi, ${ }^{6} \mathrm{M}$ Maes, ${ }^{1} \mathrm{ER}$ Delicato de Almeida, ${ }^{1} \mathrm{H}$ Kaminami Morimoto. ${ }^{1}$ University of Londrina, Department of Pathology- Clinical Analysis and Toxicology, Londrina, Brazil; ${ }^{2}$ University of Londrina, Biological Science, Londrina, Brazil; ${ }^{3}$ University of Londrina, Health Science, Londrina, Brazil; ${ }^{4}$ University of Londrina, Rheumatology, Londrina, Brazil; ${ }^{5}$ University of Londrina, Department of Internal Medicine, Londrina, Brazil; ${ }^{6}$ Deakin University, IMPACT Strategic Research Centre- School of Medicine, Geelong, Australia

\subsection{6/lupus-2017-000215.458}

Background and aims To delineate disorders in adhesion molecules in systemic lupus erythematosus (SLE) and to assess whether cortisol, nuclear autoantibody (ANA) titers and the metabolic syndrome (MetS) are associated with adhesion molecules in SLE.

Methods 48 healthy individuals and 171 SLE patients were enrolled. Disease activity was determined by SLEDAI (SLE Disease Activity Index) score. Adhesion molecules and cortisol levels were evaluated.

Results Platelet endothelial cell adhesion molecule 1 (PECAM1), Vascular cell adhesion molecule 1 (VCAM-1), E-selectin, Pselectin and Plasminogen activator inhibitor type-1 (PAI-1) were significantly higher in SLE patients. These significant differences could not be explained by the drug treatment. Mycophenolate treatment significantly decreased intercellular adhesion molecule 1 (ICAM-1) and increased E-selectin levels. Binary logistic regression analysis showed that PECAM-1 and PAI-1 predicted SLE with a sensitivity of $86.5 \%$ and a specificity of $81.3 \%$. ANA was significantly and positively associated with PECAM-1, VCAM-1, E-selectin, and PAI-1, whilst cortisol was negatively associated with PCAM-1 and ICAM-1. There were significant associations between MetS and E-selectin and PAI-1. $18.2 \%$ of the variance in SLEDAI score was explained by increased PECAM-1 values and DNA titers and the MetS.

Conclusions Our data confirm that adhesion molecules play a role in the pathophysiology of SLE and show that increased adhesion molecule levels, especially PECAM-1, can be used as an external validating criterion for the diagnosis SLE. MetS, ANA, and cortisol modulate the adhesion molecule concentrations but do not explain the increased levels in SLE Increased levels of adhesion molecules are a new drug target in SLE.

\section{SYSTEMIC LUPUS ERYTHEMATOSUS AND SEVERITY OF ILLNESS ARE ASSOCIATED WITH T HELPER 1 AND 17 CYTOKINES PROFILES TOGETHER WITH A LOWERED IL-4 PRODUCTION}

${ }^{1} \mathrm{~A}$ Name Colado Simão*, ${ }^{2} \mathrm{P}$ Macedo Guimarães, ${ }^{2} \mathrm{~B}$ Miglioranza Acavuzzi, ${ }^{2} \mathrm{D}$ Frizon Alfieri, ${ }^{3} \mathrm{~N}$ Perugini Stadtlober, ${ }^{1} \mathrm{MA}$ Batisti Lozovoy, ${ }^{1} \mathrm{EM}$ Vissoci Reiche, ${ }^{1} \mathrm{H}$ Kaminami Morimoto, ${ }^{1}$ ER Delicato de Almeida, ${ }^{4} \mathrm{~T}$ Mayumi Veiga Iriyoda, ${ }^{4} \mathrm{~N}$ Tomimura Costa, ${ }^{5}$ Dichi, ${ }^{6} \mathrm{M}$ Maes. 'University of Londrina, Department of Pathology- Clinical Analysis and Toxicology, Londrina, Brazil; ${ }^{2}$ University of Londrina, Program of Health Sciences, Londrina, Brazil; ${ }^{3}$ University of Londrina, Biological Science, Londrina, Brazil; ${ }^{4}$ University of Londrina, Department of Rheumatology, Londrina, Brazil; ${ }^{5}$ University of Londrina, Department of Internal Medicine, Londrina, Brazil; ${ }^{6}$ Deakin University, IMPACT Strategic Research CentreSchool of Medicine, Geelong, Australia

\subsection{6/lupus-2017-000215.459}

Background and aims To delineate cytokines profiles of systemic lupus erythematosus (SLE), construct prediction models for diagnosis and disease activity using those profiles, and examine how TNFB Ncol polymorphism, body mass index (BMI), vitamin $\mathrm{D}$, and cortisol influence cytokine levels in SLE.

Methods 200 SLE patients and 196 healthy controls participated in this case-control study. Plasma cytokines levels of TNF- $\alpha$, IFN- $\gamma$, IL-1 $\beta$, IL-4, IL-6, IL-10, IL-12, IL-17 were measured and cytokines profiles were computed.

Results IL-6, IL-12, IL-17, IFN- $\gamma$ and IL-10 levels were significantly higher in SLE, while IL-4 was lower in SLE. The Th1/ Th2 and Th1 + Th17/Th2 profiles were significantly higher in SLE than in healthy controls, whereas there were no significant differences in the pro-inflammatory cytokine profile (TNF $\alpha+$ IL-6 + IL-1 $\beta$ ). $90.4 \%$ of all subjects were correctly classified using Th1+Th17 profile and IL-10 (positively associated) and IL-4 (negatively associated) as predictor variables (sensitivity $=66.7 \%$ and specificity $=96.9 \%$ ). 20.9\% of the variance in the SLEDAI was predicted by the Th1+Th17/Th2 ratio, IL-10 and BMI (all positively) and proinflammatory profile (inversely associated). B1/B1 genotype is accompanied by increased IL-17 and Th17/Th2 ratio, while B1/B2 genotype is accompanied by higher IL-4 and IFN $\gamma$ values. $25-\mathrm{OH}$ vitamin $\mathrm{D}$ was inversely associated with IFN- $\gamma$ levels.

Conclusions SLE is accompanied by Th1, Th17, and Treg profile and lowered IL-4 production. Lowered vitamin D levels and B1/B1 genotype, but not cortisol or BMI, contribute to changes in cytokines profiles. Future treatments should target Th1, Th2 and Th17 profiles rather than inflammatory cytokines. 